\title{
The Odd Log-Logistic Marshall-Olkin Lindley Model for Lifetime Data
}

\author{
Morad Alizadeh \\ Department of Statistics, Faculty of Sciences,Persian Gulf University, \\ Bushehr, 75169, Iran \\ moradalizadeh78@gmail.com \\ Gamze Ozel \\ Department of Statistics, Hacettepe University \\ Ankara, 06800, Turkey \\ gamzeozl@hacettepe.edu.tr \\ Emrah Altun* \\ Department of Statistics, Hacettepe University \\ Ankara, 06800, Turkey \\ emrahaltun@hacettepe.edu.tr \\ Mousa Abdi \\ Department of Mathematics and Soft Computing \\ Higher Education Complex of Bam, Bam, Iran \\ me.abdi@bam.ac.ir \\ G.G. Hamedani \\ Department of Mathematics, Statistics and Computer Science, \\ Marquette University, USA \\ gholamhoss.hamedani@marquette.edu
}

Received 11 November 2016

Accepted 8 February 2017

\begin{abstract}
A new three parameter model called the odd log-logistic Marshall-Olkin-Lindley (OLLMO-L) distribution is proposed and studied. This distribution is symmetric, platykurtic, leptokurtic and unimodal. Various structural properties are derived, including explicit expressions for the ordinary and conditional moments, generating function and order statistics. We use maximum likelihood method to estimate the parameters of model. Simulation studies for some particular cases are also run to assess the performance of the maximum likelihood estimators. We prove empirically that the proposed distribution is a very competitive model to other classical models by means of two real data sets.
\end{abstract}

Keywords: Lindley distribution; Maximum Likelihood; Moment; Odd log-logistic; Lifetime data; Characterizations

2000 Mathematics Subject Classification: 60E05, 62E10

$\overline{{ }^{*} \text { Corresponding author. }}$

Copyright (C) 2017, the Authors. Published by Atlantis Press.

This is an open access article under the CC BY-NC license (http://creativecommons.org/licenses/by-nc/4.0/). 


\section{Introduction}

The Lindley distribution is a very well-known distribution that has been extensively used over the past decades for modeling data in reliability, biology, insurance, finance and lifetime analysis. The Lindley distribution was introduced by Lindley (1958) to analyze failure time data. The motivation for introducing the Lindley distribution arises from its ability to model failure time data with increasing, decreasing, unimodal and bathtub shaped hazard rates. This distribution represents a good alternative to the exponential failure time distribution which does not not exhibit unimodality and bathtub shaped failure rates.

The need for extended forms of the Lindley distribution arises in many applied areas. The emergence of such distributions in the statistics literature is only very recent. For some extended forms of the Lindley distribution and their applications, the reader is referred to Kumaraswamy Lindley (Cakmakyapan \& Ozel, 2014), beta odd log-logistic Lindley (Cordeiro et al., 2015), generalized Lindley (Nadarajah et al., 2011), quasi Lindley distribution (Shanker \& Mishra, 2013), inverse Lindley (Sharma et al., 2015) and power Lindley (Ghitany et al. 2013).

The cumulative distribution function (cdf) and probability density function (pdf) of the Lindley distribution are given, respectively, by

$$
g(x ; \lambda)=\frac{\lambda^{2}}{1+\lambda}(1+x) \mathrm{e}^{-\lambda \mathrm{x}}, \mathrm{x}>0, \quad \lambda>0
$$

and

$$
G(x ; \lambda)=1-\left(1+\frac{\lambda x}{1+\lambda}\right) \mathrm{e}^{-\lambda x}, x>0, \quad \lambda>0 .
$$

Note that this distribution is a mixture of exponential $(\lambda)$ and gamma $(2, \lambda)$ distributions with respective mixing proportions $\frac{\lambda}{1+\lambda}$ and $\frac{1}{1+\lambda}$. The One parameter Lindley distribution does not provide enough flexibility for analyzing different types of lifetime data. Hence, it will be useful to consider other alternatives to this distribution for modelling purposes. Therefore, the goal of the present study is to introduce a new distribution using the Lindley distribution as the baseline distribution.

Gleaton and Lynch $(2006,2010)$ look at groups of transformations that divide the collection of life distributions into equivalent classes. The one related to (1.3) (below) is given by transformations given by the composition of proportional odds transformation with the generalized log-logistic transformation and they refer to the family as an extended generalized log-logistic family. This family was called later the odd log-logistic Marshall-Olkin (OLLMO-G) family of distributions. An equivalence class is the family given by (1.3) with $\xi$ fixed and $\alpha, \beta>0$. When $\alpha=1$ and $\beta>0$, Equation (1.3) reduces to the original Marshall-Olkin family of distributions introduced by Marshall and Olkin (1997). The pdf and cdf of OLLMO-G family are given, respectively, by

$$
F(x ; \alpha, \beta, \xi)=\frac{G(x ; \xi)^{\alpha}}{G(x ; \xi)^{\alpha}+\beta \bar{G}(x ; \xi)^{\alpha}}, \alpha, \beta>0,
$$

and pdf

$$
f(x ; \alpha, \beta, \xi)=\frac{\alpha \beta g(x ; \xi) G(x ; \xi)^{\alpha-1} \bar{G}(x ; \xi)^{\alpha-1}}{\left[G(x ; \xi)^{\alpha}+\beta \bar{G}(x ; \xi)^{\alpha}\right]^{2}},
$$


where $\bar{G}(x ; \xi)=1-G(x ; \xi)$ and $G(x ; \xi)$ is a baseline cdf which depends on a parameter vector $\xi$. For $\beta=1$, the parameter $\alpha$ in (1.3) represents the quotient of the log odds ratio for the generated and baseline distributions as follows:

$$
\alpha=\frac{\log \left[\frac{F(x ; \alpha, \xi)}{\bar{F}(x ; \alpha, \xi)}\right]}{\log \left[\frac{G(x ; \xi)}{\bar{G}(x ; \xi)}\right]} .
$$

Based on the OLLMO-G family of Gleaton and Lynch (2010), we propose a new distribution called OLLMO-L by inserting (1.2) in (1.3), having cdf

$$
F(x ; \alpha, \beta, \lambda)=\frac{\left[1-\left(1+\frac{\lambda}{1+\lambda} x\right) \mathrm{e}^{-\lambda x}\right]^{\alpha}}{\left[1-\left(1+\frac{\lambda}{1+\lambda} x\right) \mathrm{e}^{-\lambda x}\right]^{\alpha}+\beta\left[\left(1+\frac{\lambda}{1+\lambda} x\right) \mathrm{e}^{-\lambda x}\right]^{\alpha}}
$$

Then, the corresponding pdf of the OLLMO-L distribution is given by

$$
f(x ; \alpha, \beta, \lambda)=\frac{\alpha \beta \lambda^{2}(1+x)\left(1+\frac{\lambda}{1+\lambda} x\right)^{\alpha-1} \mathrm{e}^{-\alpha \lambda x}\left[1-\left(1+\frac{\lambda}{1+\lambda} x\right) \mathrm{e}^{-\lambda x}\right]^{\alpha-1}}{(1+\lambda)\left\{\left[1-\left(1+\frac{\lambda}{1+\lambda} x\right) \mathrm{e}^{-\lambda x}\right]^{\alpha}+\beta\left[\left(1+\frac{\lambda}{1+\lambda} x\right) \mathrm{e}^{-\lambda x}\right]^{\alpha}\right\}^{2}} .
$$

A random variable $X$ with pdf (1.6) is denoted by $X \sim \operatorname{OLLMO}-\mathrm{L}(\alpha, \beta, \lambda)$. The OLLMO-L distribution is more flexible than the Lindley distribution and allows for greater flexibility of the tails. Plots for the density function of OLLMO-L for several parameter values are displayed in Figures 1. As seen from Figures 1, the density function can take various forms depending on the parameter values such as unimodal, symmetric, skewed, and monotonically decreasing shapes appear to be possible. Some special models of (1.6) are given in Table 1.

Table 1. Some special models of the OLLMO-L distribution

\begin{tabular}{|c|c|c|}
\hline$\alpha$ & $\beta$ & Reduced distribution \\
\hline 1 & 1 & Lindley \\
\hline- & 1 & Odd log-logistic Lindley \\
\hline 1 & - & Marshal-Olkin Lindley \\
\hline
\end{tabular}

The rest of the paper is organized as follows. In Section 2, we provide a physical interpretation of the OLLMO-L distribution. We also discuss the mathematical properties of the proposed distribution, including survival and hazard rate functions, quantile functions, pdf and cdf expansions, moments and moment generating functions and asymptotic. Order statistics is presented in Section 3. Section 4 is devoted to certain characterizations of OLLMO-L distribution. Inference and estimation by the method of maximum likelihood and an explicit expression for the observed information matrix are presented in Section 5. Section 6 presents a simulation study. Two applications to real data set is considered in Section 7. Finally, Section 8 offers some concluding remarks. 

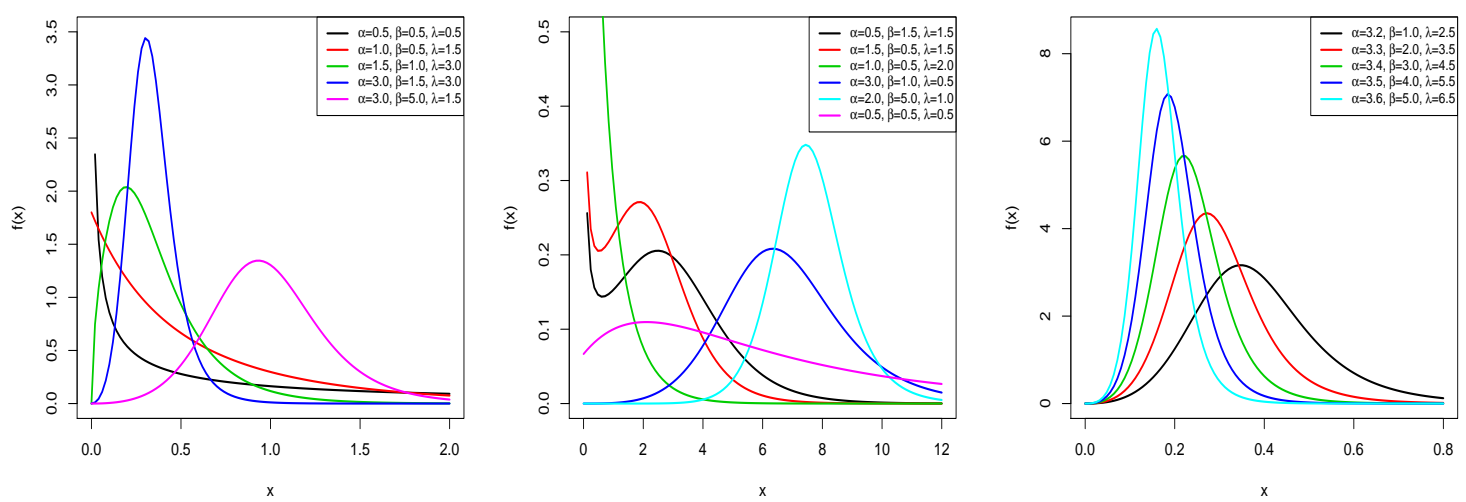

Fig. 1. Plots of the OLLMO-L density function for some parameter values.

\section{Main Properties}

\subsection{Survival and Hazard Rate Functions}

Central role is played in the reliability theory by the quotient of the pdf and survival function. We obtain the survival function corresponding to (1.5) as

$$
S(x ; \alpha, \beta, \lambda)=1-\frac{\left[1-\left(1+\frac{\lambda}{1+\lambda} x\right) \mathrm{e}^{-\lambda x}\right]^{\alpha}}{\left[1-\left(1+\frac{\lambda}{1+\lambda} x\right) \mathrm{e}^{-\lambda x}\right]^{\alpha}+\beta\left[\left(1+\frac{\lambda}{1+\lambda} x\right) \mathrm{e}^{-\lambda x}\right]^{\alpha}} .
$$

In reliability studies, the hazard rate function (hrf) is an important characteristic and fundamental to the design of safe systems in a wide variety of applications. Therefore, we discuss these properties for the OLLMO-L distribution. The hrf of X takes the form

$$
h(x ; \alpha, \beta, \lambda)=\frac{\alpha \beta \lambda^{2}(1+x)\left[1-\left(1+\frac{\lambda}{1+\lambda} x\right) \mathrm{e}^{-\lambda x}\right]^{\alpha-1}}{(1+\lambda)\left(1+\frac{\lambda}{1+\lambda} x\right)\left\{\left[1-\left(1+\frac{\lambda}{1+\lambda} x\right) \mathrm{e}^{-\lambda x}\right]^{\alpha}+\beta\left[\left(1+\frac{\lambda}{1+\lambda} x\right) \mathrm{e}^{-\lambda x}\right]^{\alpha}\right.} .
$$

Plots for the hrfs of the OLLMO-L distribution for several parameter values are displayed in Figures 2. Figures 2 shows that the hrf of the OLLMO-L distribution can have very flexible shapes, such as increasing, decreasing, upside-down, bathtub. It is evident that the OLLMO-L distribution is much more flexible than the Lindley distribution, i.e. the additional parameter $\alpha>0$ allows for a high degree of flexibility of the OLLMO-L distribution. This attractive flexibility makes the hrf of the OLLMO-L useful and suitable for non-monotone empirical hazard behaviour which are more likely to be encountered or observed in real life situations. 

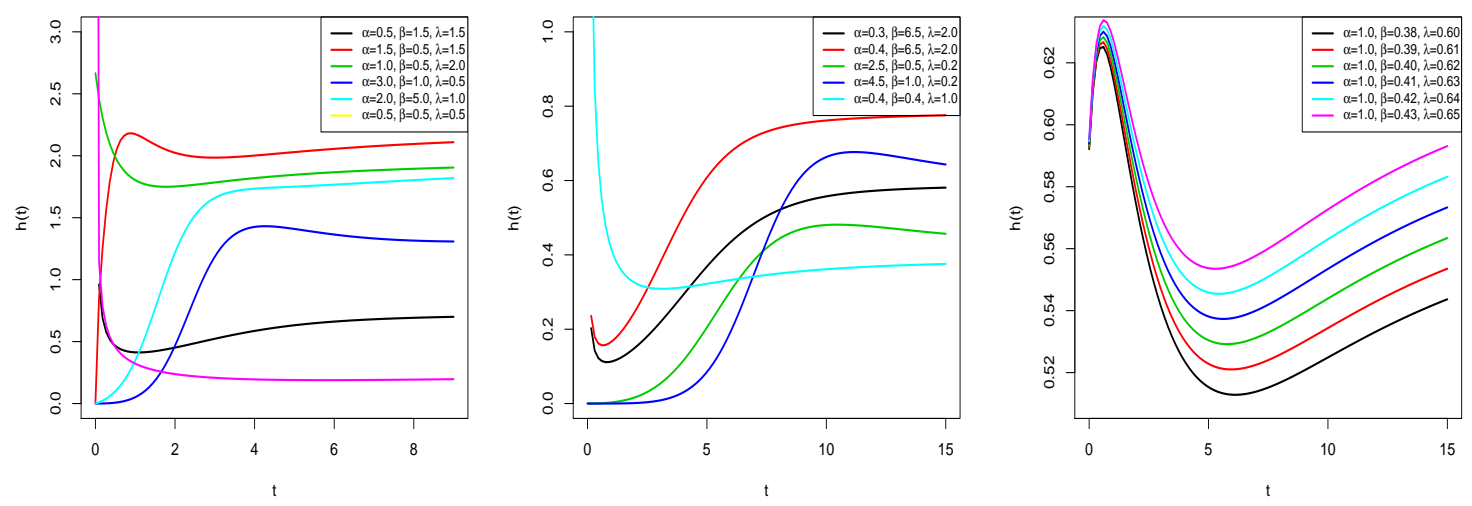

Fig. 2. Plots of the OLLMO-L hrfs for some parameter values.

\subsection{Quantile Function}

Quantile functions are in widespread use in statistics and often find representations in terms of lookup tables for key percentiles. Let $X \sim \operatorname{OLLMO}-\mathrm{L}(\alpha, \beta, \lambda)$ random variable. The quantile function, say $Q(p)$, defined by $F[Q(p)]=p$, is the root of the equation

$$
[1+\lambda+\lambda Q(p)] \mathrm{e}^{-\lambda Q(p)}=\frac{(1+\lambda)(1-p)^{\frac{1}{\alpha}}}{(\beta p)^{\frac{1}{\alpha}}+(1-p)^{\frac{1}{\alpha}}},
$$

for $0<p<1$. Substituting $Z(p)=-1-\lambda-\lambda Q(p)$, one can rewrite (2.3) as

$$
Z(p) \mathrm{e}^{Z(p)}=\frac{-(1+\lambda)(1-p)^{\frac{1}{\alpha}} \mathrm{e}^{-1-\lambda}}{(\beta p)^{\frac{1}{\alpha}}+(1-p)^{\frac{1}{\alpha}}} .
$$

Hence, the solution $Z(p)$ is

$$
Z(p)=W\left[\frac{-(1+\lambda)(1-p)^{\frac{1}{\alpha}} \mathrm{e}^{-1-\lambda}}{(\beta p)^{\frac{1}{\alpha}}+(1-p)^{\frac{1}{\alpha}}}\right],
$$

where $W[$.$] is the Lambert function (Corless et al. 1996). Inserting (2.5) into Z(p)=-1-\lambda-Q(p)$ gives

$$
Q(p)=-1-\frac{1}{\lambda}-\frac{1}{\lambda} W\left[\frac{-(1+\lambda)(1-p)^{\frac{1}{\alpha}} \mathrm{e}^{-1-\lambda}}{(\beta p)^{\frac{1}{\alpha}}+(1-p)^{\frac{1}{\alpha}}}\right] .
$$

The particular case of (2.6) for $\alpha=\beta=1$ has been derived recently by Jodrá (2010). Here, we propose two different algorithms for generating random data from the OLLMO-L distribution.

(a) The first algorithm is based on generating random data from the Lindley distribution using the exponential gamma mixture.

\section{Algorithm 1 (Mixture form of the Lindley distribution)}

- Generate $U_{i} \sim \operatorname{Uniform}(0,1), \quad i=1, \ldots, n$; 
- Generate $V_{i} \sim \operatorname{Exponential}(\lambda), \quad i=1, \ldots, n$;

- Generate $W_{i} \sim \operatorname{Gamma}(2, \lambda), \quad i=1, \ldots, n$;

- If $\frac{\left(\beta U_{i}\right)^{\frac{1}{\alpha}}}{\left(\beta U_{i}\right)^{\frac{1}{\alpha}}+\left(1-U_{i}\right)^{\frac{1}{\alpha}}} \leq \frac{\lambda}{1+\lambda}$ set $X_{i}=V_{i}$, otherwise, set $X_{i}=W_{i}, \quad i=1, \ldots, n$.

(b) The second algorithm is based on generating random data from the inverse cdf in (1.5) of OLLMO-L distribution.

Algorithm 2 (Inverse cdf)

- Generate $U_{i} \sim$ Uniform $(0,1), \quad i=1, \ldots, n$;

- Set

$$
X_{i}=\left\{-1-\frac{1}{\lambda}-\frac{1}{\lambda} W\left[\frac{-(1+\lambda)\left(1-U_{i}\right)^{\frac{1}{\alpha}} \mathrm{e}^{-1-\lambda}}{\left(\beta U_{i}\right)^{\frac{1}{\alpha}}+\left(1-U_{i}\right)^{\frac{1}{\alpha}}}\right]\right\}, \quad i=1, \ldots, n
$$

\subsection{Expansions for the Cumulative and Density Functions}

In this subsection, we provide alternative mixture representations for the pdf and cdf of OLLMO-L distribution. Despite the fact that the pdf and cdf of OLLMO-L require mathematical functions that are widely available in modern statistical packages, frequently analytical and numerical derivations take advantage of power series for the pdf. Some useful expansions for (1.5) can be derived by using the concept of power series. We obtain the pdf of OLLMO-L as

$$
\left\{1-\left(1+\frac{\lambda x}{1+\lambda}\right) \mathrm{e}^{-\lambda x}\right\}^{\alpha}=\sum_{k=0}^{\infty} a_{k}\left\{1-\left(1+\frac{\lambda x}{1+\lambda}\right) \mathrm{e}^{-\lambda x}\right\}^{k},
$$

where $a_{k}=\sum_{i=k}^{\infty}(-1)^{i+k}\left(\begin{array}{c}\alpha \\ i\end{array}\right)\left(\begin{array}{l}i \\ k\end{array}\right)$ and

$$
\left[1-\left(1+\frac{\lambda}{1+\lambda} x\right) \mathrm{e}^{-\lambda x}\right]^{\alpha}+\beta\left[\left(1+\frac{\lambda}{1+\lambda} x\right) \mathrm{e}^{-\lambda x}\right]^{\alpha}=\sum_{k=0}^{\infty} b_{k}\left\{1-\left(1+\frac{\lambda x}{1+\lambda}\right) \mathrm{e}^{-\lambda x}\right\}^{k},
$$

where $b_{k}=a_{k}+\beta(-1)^{k}\left(\begin{array}{c}\alpha \\ i\end{array}\right)$.

Identity (2.7) is a consequence of the generalized binomial expansion of

$$
(1-\Delta)^{\alpha}=\sum_{k=0}^{\infty}\left(\begin{array}{l}
\alpha \\
k
\end{array}\right)(-1)^{k} \Delta^{k}
$$

where $\left(\begin{array}{l}\alpha \\ k\end{array}\right)=\frac{\alpha(\alpha-1) \cdots(\alpha-k+1)}{k !}$ and the binomial expansion of

$$
\Delta^{k}=\sum_{j=0}^{k}\left(\begin{array}{l}
k \\
j
\end{array}\right)(-1)^{j}(1-\Delta)^{j}
$$

Substituting the latter expansion into the former one, interchanging the order of summation and replacing $\Delta=\left(1+\frac{\lambda x}{1+\lambda}\right) \mathrm{e}^{-\lambda x}$ gives (2.7). 
Then we can write

$$
F(x)=\frac{\sum_{k=0}^{\infty} a_{k}\left\{1-\left(1+\frac{\lambda x}{1+\lambda}\right) \mathrm{e}^{-\lambda x}\right\}^{k}}{\sum_{k=0}^{\infty} b_{k}\left\{1-\left(1+\frac{\lambda x}{1+\lambda}\right) \mathrm{e}^{-\lambda x}\right\}^{k}}=\sum_{k=0}^{\infty} c_{k}\left\{1-\left(1+\frac{\lambda x}{1+\lambda}\right) \mathrm{e}^{-\lambda x}\right\}^{k},
$$

where $c_{0}=\frac{a_{0}}{b_{0}}$ and for $k \geq 1$,

$$
c_{k}=b_{0}^{-1}\left[a_{k}-b_{0}^{-1} \sum_{r=1}^{k} b_{r} c_{k-r}\right]
$$

We can write

$$
F(x)=\sum_{k=0}^{\infty} c_{k} G_{k}(x)
$$

where $G_{k}(x)$ denotes the cdf of generalized Lindley (exponentiated Lindley) distribution with parameters $\lambda$ and $k$.

Now, by differentiation (2.11), the pdf of $\mathrm{X}$ can be expressed as

$$
f(x)=\sum_{k=0}^{\infty} c_{k+1} g_{k+1}(x),
$$

where $g_{k+1}(x)$ denotes the pdf of generalized Lindley (exponentiated Lindley) distribution with parameters $\lambda$ and $k$. Several properties of the OLLMO-L distribution can be obtained from pdf and cdf expansions given in (2.11) and (2.12), respectively.

\subsection{Moments and Moment Generating Function}

Some of the most important features and characteristics of a distribution can be studied through moments (e.g. tendency, dispersion, skewness and kurtosis). We obtain ordinary moments and the moment generating function of the OLLMO-L distribution. Nadarajah et al. (2011) defined and computed

$$
A(a, b, c, \delta)=\int_{0}^{\infty} x^{c}(1+x)\left[1-\left(1+\frac{b x}{b+1}\right) \mathrm{e}^{-b x}\right]^{a-1} \mathrm{e}^{-\delta x} d x
$$

which can be used to produce ordinary moments $\left(\mu_{r}^{\prime}\right)$. Then, we have

$$
A(a, b, c, \delta)=\sum_{l=0}^{\infty} \sum_{r=0}^{l} \sum_{s=0}^{r+1}\left(\begin{array}{c}
a-1 \\
l
\end{array}\right)\left(\begin{array}{c}
l \\
r
\end{array}\right)\left(\begin{array}{c}
r+1 \\
s
\end{array}\right) \frac{(-1)^{l} b^{r} \Gamma(s+c+1)}{(1+b)^{l}(b l+\delta)^{c+s+1}}
$$

From equations (2.11) and (2.12), we obtain

$$
\mu_{r}^{\prime}=E\left[X^{r}\right]=\frac{\lambda^{2}}{1+\lambda} \sum_{k=0}^{\infty}(k+1) c_{k+1} A(k+1, \lambda, r, \lambda) .
$$

The ordinary moments of the OLLMO-L distribution can be calculated directly from (2.15). 
We now provide a formula for the conditional moments of the OLLMO-L distribution. Nadarajah et al. (2011) defined and computed the following equation for the conditional moments

$$
L(a, b, c, \delta, t)=\int_{t}^{\infty} x^{c}(1+x)\left[1-\left(1+\frac{b x}{b+1}\right) \mathrm{e}^{-b x}\right] \mathrm{e}^{-\delta x} d x .
$$

Using generalized binomial expansion, we have

$$
L(a, b, c, \delta, t)=\sum_{l=0}^{\infty} \sum_{r=0}^{l} \sum_{s=0}^{r+1}\left(\begin{array}{c}
a-1 \\
l
\end{array}\right)\left(\begin{array}{c}
l \\
r
\end{array}\right)\left(\begin{array}{c}
r+1 \\
s
\end{array}\right) \frac{(-1)^{l} b^{r} \Gamma(s+c+1,(b l+\delta) t)}{(1+b)^{l}(b l+\delta)^{c+s+1}},
$$

where

$$
\Gamma(a, x)=\int_{x}^{\infty} t^{a-1} \mathrm{e}^{-t} d t
$$

denotes the incomplete gamma function. From (2.12) and (2.17), we obtain

$$
\mu_{r}^{\prime}(t)=E\left[X^{r} \mid X>t\right]=\frac{\lambda^{2}}{1+\lambda} \sum_{k=0}^{\infty}(k+1) c_{k+1} L(k+1, \lambda, r, \lambda, t) .
$$

The incomplete moments of the OLLMO-L distribution can be calculated directly from (2.19).

The moment generating function (mgf) of a random variable provides the basis for an alternative route to analytical results compared with working directly with its pdf and cdf. Using (2.12) and (2.14), we obtain

$$
M_{X}(t)=E\left[\mathrm{e}^{\mathrm{tX}}\right]=\frac{\lambda^{2}}{1+\lambda} \sum_{k=0}^{\infty}(k+1) c_{k+1} A(k+1, \lambda, 0, \lambda-t) .
$$

Remark 2.1. The central moments $\left(\mu_{n}\right)$ and cumulants $\left(\kappa_{n}\right)$ of $X$ are easily obtained from (2.15) as

$$
\mu_{n}=\sum_{k=0}^{n}(-1)^{k}\left(\begin{array}{l}
n \\
k
\end{array}\right) \mu_{1}^{\prime k} \mu_{n-k}^{\prime} \quad \text { and } \quad \kappa_{n}=\mu_{n}^{\prime}-\sum_{k=1}^{n-1}\left(\begin{array}{l}
n-1 \\
k-1
\end{array}\right) \kappa_{k} \mu_{n-k}^{\prime},
$$

respectively, where $\kappa_{1}=\mu_{1}^{\prime}$. Thus, $\kappa_{2}=\mu_{2}^{\prime}-\mu_{1}^{\prime 2}, \kappa_{3}=\mu_{3}^{\prime}-3 \mu_{2}^{\prime} \mu_{1}^{\prime}+2 \mu_{1}^{\prime 3}$, etc.

\subsection{Asymptotic}

Let $X \sim$ OLLMO-L $(\alpha, \beta, \lambda)$, the asymptotic of (1.5), (1.6) and (2.2) as $x \rightarrow 0$ are given by

$$
\begin{aligned}
& F(x) \sim \frac{(\lambda x)^{\alpha}}{\beta} \text { as } x \rightarrow 0, \\
& f(x) \sim \frac{\alpha \lambda^{\alpha} x^{\alpha-1}}{\beta} \text { as } x \rightarrow 0, \\
& h(x) \sim \frac{\alpha \lambda^{\alpha} x^{\alpha-1}}{\beta} \text { as } x \rightarrow 0 .
\end{aligned}
$$

The asymptotic of (1.5), (1.6) and (2.2) as $x \rightarrow \infty$ are given by 


$$
\begin{aligned}
& f(x) \sim \alpha \beta \lambda\left(\frac{\lambda}{1+\lambda}\right)^{\alpha} x^{\alpha-1} \mathrm{e}^{-\alpha \lambda x} \text { as } x \rightarrow \infty, \\
& 1-F(x) \sim \beta\left(\frac{\lambda}{1+\lambda}\right)^{\alpha} x^{\alpha} \mathrm{e}^{-\alpha \lambda x} \text { as } x \rightarrow \infty, \\
& h(x) \sim \frac{\alpha \beta \lambda}{x} \text { as } x \rightarrow \infty .
\end{aligned}
$$

\section{Order statistics}

Order statistics make their appearance in many areas of statistical theory and practice. Suppose $X_{1}, \ldots, X_{n}$ is a random sample from the OLLMO-L distribution. Let $X_{i: n}$ denote the $i$ th order statistic. The pdf of $X_{i: n}$ can be expressed as

$$
f_{i: n}(x)=K \sum_{j=0}^{n-i}(-1)^{j}\left(\begin{array}{c}
n-i \\
j
\end{array}\right)\left[\sum_{r=0}^{\infty} c_{r+1}(r+1) G(x)^{r} g(x)\right]\left[\sum_{k=0}^{\infty} c_{k} G(x)^{k}\right]^{j+i-1},
$$

where $K=n ! /[(i-1) !(n-i) !]$. Using a result of Gradshteyn and Ryzhik (2000, Section 0.314) for a power series raised to a positive integer, we obtain

$$
\left[\sum_{k=0}^{\infty} c_{k} G(x)^{k}\right]^{j+i-1}=\sum_{k=0}^{\infty} e_{j+i-1, k} G(x)^{k},
$$

where $e_{j+i-1,0}=c_{0}^{j+i-1}$ and, for $k \geq 1$,

$$
e_{j+i-1, k}=\left(k c_{0}\right)^{-1} \sum_{q=1}^{k}[q(j+i)-k] c_{q} e_{j+i-1, k-q} .
$$

Setting $d_{r}=(r+1) c_{r+1}$ and using a result of Gradshteyn and Ryzhik (2000, Section 0.316) for multiplying two power series, we have

$$
\begin{aligned}
f_{i: n}(x) & =K \sum_{j=0}^{n-i}(-1)^{j}\left(\begin{array}{c}
n-i \\
j
\end{array}\right) g(x)\left[\sum_{r=0}^{\infty} d_{r} G(x)^{r}\right]\left[\sum_{k=0}^{\infty} e_{j+i-1, k} G(x)^{k}\right] \\
& =K \sum_{j=0}^{n-i}(-1)^{j}\left(\begin{array}{c}
n-i \\
j
\end{array}\right) g(x) \sum_{k=0}^{\infty} e_{k}^{*} G(x)^{k},
\end{aligned}
$$

where $e_{k}^{*}=\sum_{q=0}^{k} e_{j+i-1, q} d_{k-q}$. Hence, we can write

$$
f_{i: n}(x)=\sum_{k=0}^{\infty} s_{k} h_{k+1}(x)
$$

where (for $k \geq 0$ )

$$
s_{k}=\frac{k}{k+1} \sum_{j=0}^{n-i}(-1)^{j}\left(\begin{array}{c}
n-i \\
j
\end{array}\right) e_{k}^{*}
$$


Equation (3.1) is the main result of this section. It reveals that the pdf of the OLLMO-L an order statistic is a triple linear combination of exponentiated power Lindley distributions. Therefore, several mathematical quantities of these order statistics like ordinary and incomplete moments, factorial moments, mgf, mean deviations and others can be derived using this result.

\section{Characterizations}

In this section we present certain characterizations of OLLMO-L distribution. These characterizations are in terms of: $(i)$ a simple relation between two truncated moments; (ii) the hazard function and (iii) the reverse hazard function. One of the advantages of characterization $(i)$ is that the cdf is not required to have a closed form.

We present our characterizations $(i)-($ iii $)$ in three subsections.

\subsection{Characterizations based on ratio of two truncated moments}

In this subsection we present characterizations of OLLMO-L distribution in terms of a simple relationship between two truncated moments. This characterization result employs a theorem due to Glänzel [1], see Theorem A,1 of Appendix A. Note that the result holds also when the interval $H$ is not closed. Moreover, as mentioned above, it could also be applied when the cdf $F$ does not have a closed form. As shown in [2], this characterization is stable in the sense of weak convergence.

Proposition 4.1. Let $X: \Omega \rightarrow(0, \infty)$ be a continuous random variable and let $q_{1}(x)=$ $e^{(\alpha-1) \lambda x}\left(1+\frac{\lambda}{1+\lambda} x\right)^{1-\alpha}\left\{\left[1-\left(1+\frac{\lambda}{1+\lambda} x\right) e^{-\lambda x}\right]^{\alpha}+\beta\left[\left(1+\frac{\lambda}{1+\lambda} x\right) e^{-\lambda x}\right]^{\alpha}\right\}^{2}$ and $q_{2}(x)=q_{1}(x)$ $\left[1-\left(1+\frac{\lambda}{1+\lambda} x\right) e^{-\lambda x}\right]^{\alpha}$ for $x>0$. The random variable $X$ has $p d f$ (1.6) if and only if the function $\eta$ defined in Theorem A.1 has the form

$$
\eta(x)=\frac{1}{2}\left\{1+\left[1-\left(1+\frac{\lambda}{1+\lambda} x\right) e^{-\lambda x}\right]^{\alpha}\right\}, \quad x>0 .
$$

Proof. Let $X$ be a random variable with pdf (1.6), then

$$
(1-F(x)) E\left[q_{1}(x) \mid X \geq x\right]=\beta\left\{1-\left[1-\left(1+\frac{\lambda}{1+\lambda} x\right) e^{-\lambda x}\right]^{\alpha}\right\}, \quad x>0
$$

and

$$
(1-F(x)) E\left[q_{2}(x) \mid X \geq x\right]=\frac{\beta}{2}\left\{1-\left[1-\left(1+\frac{\lambda}{1+\lambda} x\right) e^{-\lambda x}\right]^{2 \alpha}\right\}, \quad x>0,
$$

and finally

$$
\eta(x) q_{1}(x)-q_{2}(x)=\frac{1}{2} q_{1}(x)\left\{1-\left[1-\left(1+\frac{\lambda}{1+\lambda} x\right) e^{-\lambda x}\right]^{\alpha}\right\}>0 \text { for } x>0 .
$$

Conversely, if $\eta$ is given as above, then 


$$
s^{\prime}(x)=\frac{\eta^{\prime}(x) q_{1}(x)}{\eta(x) q_{1}(x)-q_{2}(x)}=\frac{\alpha \lambda^{2}(1+x) e^{-\lambda x}\left[1-\left(1+\frac{\lambda}{1+\lambda} x\right) e^{-\lambda x}\right]^{\alpha-1}}{(1+\lambda)\left\{1-\left[1-\left(1+\frac{\lambda}{1+\lambda} x\right) e^{-\lambda x}\right]^{\alpha}\right\}} \quad x>0,
$$

and hence

$$
s(x)=-\log \left\{1-\left[1-\left(1+\frac{\lambda}{1+\lambda} x\right) e^{-\lambda x}\right]^{\alpha}\right\}, x>0 .
$$

Now, in view of Theorem A.1, $X$ has density (1.6).

Corollary 4.1. Let $X: \Omega \rightarrow(0, \infty)$ be a continuous random variable and let $q_{1}(x)$ be as in Proposition 4.1. The pdf of $X$ is (1.6) if and only if there exist functions $q_{2}$ and $\eta$ defined in Theorem A.1 satisfying the differential equation

$$
\frac{\eta^{\prime}(x) q_{1}(x)}{\eta(x) q_{1}(x)-q_{2}(x)}=\frac{\alpha \lambda^{2}(1+x) e^{-\lambda x}\left[1-\left(1+\frac{\lambda}{1+\lambda} x\right) e^{-\lambda x}\right]^{\alpha-1}}{(1+\lambda)\left\{1-\left[1-\left(1+\frac{\lambda}{1+\lambda} x\right) e^{-\lambda x}\right]^{\alpha}\right\}}, \quad x>0
$$

The general solution of the differential equation in Corollary 4.1 is

$\eta(x)=\left\{1-\left[1-\left(1+\frac{\lambda}{1+\lambda} x\right) e^{-\lambda x}\right]^{\alpha}\right\}^{-1}\left[\begin{array}{c}-\int \frac{\alpha \lambda^{2}}{(1+\lambda)}(1+x) e^{-\lambda x} \times \\ {\left[1-\left(1+\frac{\lambda}{1+\lambda} x\right) e^{-\lambda x}\right]^{\alpha-1}\left(q_{1}(x)\right)^{-1} q_{2}(x)+D}\end{array}\right]$,

where $D$ is a constant. Note that a set of functions satisfying the above differential equation is given in Proposition 4.1 with $D=\frac{1}{2}$. However, it should be also noted that there are other triplets $\left(q_{1}, q_{2}, \eta\right)$ satisfying the conditions of Theorem A.1.

\subsection{Characterization based on hazard function}

It is known that the hazard function, $h_{F}$, of a twice differentiable distribution function, $F$, satisfies the first order differential equation

$$
\frac{f^{\prime}(x)}{f(x)}=\frac{h_{F}^{\prime}(x)}{h_{F}(x)}-h_{F}(x)
$$

For many univariate continuous distributions, this is the only characterization available in terms of the hazard function. The following characterization establish a non-trivial characterization of OLLMO-L distribution, when $\alpha=1$, which is not of the above trivial form.

Proposition 4.2. Let $X: \Omega \rightarrow(0, \infty)$ be a continuous random variable. The $p d f$ of $X$ for $\alpha=1$, is (1.6) if and only if its hazard function $h_{F}(x)$ satisfies the differential equation 


$$
\begin{array}{r}
h_{F}^{\prime}(x)-(1+x)^{-1} h_{F}(x)= \\
1+\lambda \\
\frac{e^{\lambda x}}{d x}\left\{\frac{d}{\left(1+\frac{\lambda}{1+\lambda} x\right) e^{\lambda x}+(\beta-1)\left(1+\frac{\lambda}{1+\lambda} x\right)^{2}}\right\}, x>0 .
\end{array}
$$

Proof. If $X$ has pdf (1.6), then clearly the above differential equation holds. Now, this differential equation holds, then

$$
\frac{d}{d x}\left\{(1+x)^{-1} h_{F}(x)\right\}=\frac{\lambda^{2}}{1+\lambda} \frac{d}{d x}\left\{\frac{\left(1+\frac{\lambda}{1+\lambda} x\right)^{-1}}{1+(\beta-1)\left(1+\frac{\lambda}{1+\lambda} x\right) e^{-\lambda x}}\right\}, x>0
$$

from which, we obtain

$$
h_{F}(x)=\frac{\lambda^{2}}{1+\lambda}\left\{\frac{(1+x)\left(1+\frac{\lambda}{1+\lambda} x\right)^{-1}}{1+(\beta-1)\left(1+\frac{\lambda}{1+\lambda} x\right) e^{-\lambda x}}\right\}, x>0,
$$

which is the hazard function of OLLMO-L distribution for $\alpha=1$.

Remark 4.1. for $\alpha=\beta=1$, we have a simple differential equation in terms of the hazard function

$$
h_{F}^{\prime}(x)-(1+x)^{-1} h_{F}(x)=-\frac{\lambda^{3}(1+x)}{(1+\lambda+\lambda x)^{2}}, \quad x>0 .
$$

\subsection{Characterizations in terms of the reverse hazard function}

The reverse hazard function, $r_{F}$, of a twice differentiable distribution function, $F$, is defined as

$$
r_{F}(x)=\frac{f(x)}{F(x)}, x \in \text { support of } F .
$$

This subsection deals with the characterization of OLLMO-L distribution, for $\alpha=1$, based on the reverse hazard function.

Proposition 4.3. Let $X: \Omega \rightarrow(0, \infty)$ be a continuous random variable. The random variable $X$ has $p d f$ (1.6) if and only if its reverse hazard function $r_{F}(x)$ satisfies the following differential equation

$$
\begin{aligned}
& r_{F}^{\prime}(x)-(1+x)^{-1} r_{F}(x) \\
& =\frac{\beta \lambda^{2}}{1+\lambda}(1+x) \frac{d}{d x}\left\{\frac{1}{\left[e^{\lambda x}-\left(1+\frac{\lambda}{1+\lambda} x\right)\right]\left[1+(\beta-1)\left(1+\frac{\lambda}{1+\lambda} x\right) e^{-\lambda x}\right]}\right\}, \quad x>0 .
\end{aligned}
$$

Proof. Is similar to that of Proposition 4.2. 


\section{Estimation}

We obtain the maximum likelihood estimates (MLEs) of the parameters of the OLLMO-L distribution from complete samples only. Let $x_{1}, \ldots, x_{n}$ be a random sample of size $n$ from the OLLMO-L $(\alpha, \beta, \lambda)$ distribution. The log-likelihood function for the vector of parameters $\theta=(\alpha, \beta, \lambda)^{T}$ can be written as

$$
\begin{aligned}
l(\theta) & =n \log \left(\frac{\alpha \beta \lambda^{2}}{1+\lambda}\right)+\sum_{i=1}^{n} \log \left(1+x_{i}\right)-\lambda \sum_{i=1}^{n} x_{i}+(\alpha-1) \sum_{i=1}^{n} \log \left[q_{i}\left(1-q_{i}\right)\right] \\
& -2 \sum_{i=1}^{n} \log \left[q_{i}^{\alpha}+\beta\left(1-q_{i}\right)^{\alpha}\right]
\end{aligned}
$$

where $q_{i}=1-\left(1+\frac{\lambda}{1+\lambda} x_{i}\right) \mathrm{e}^{-\lambda \mathrm{x}_{\mathrm{i}}}$ is a transformed observation.

The log-likelihood can be maximized either directly by using the SAS (Procedure NLMixed) or the MaxBFGS routine in the matrix programming language Ox (see Doornik, 2007) or by solving the nonlinear likelihood equations obtained by differentiating (5.1) simultaneously. The components of the score vector $U(\theta)$ are given by

$$
\begin{aligned}
U_{\alpha}(\theta)= & \frac{n}{\alpha}+\sum_{i=1}^{n} \log \left[q_{i}\left(1-q_{i}\right)\right]-2 \sum_{i=1}^{n} \frac{q_{i}^{\alpha} \log \left(q_{i}\right)+\beta\left(1-q_{i}\right)^{\alpha} \log \left(1-q_{i}\right)}{q_{i}^{\alpha}+\beta\left(1-q_{i}\right)^{\alpha}}, \\
U_{\beta}(\theta)= & \frac{n}{\beta}-2 \sum_{i=1}^{n} \frac{\left(1-q_{i}\right)^{\alpha}}{q_{i}^{\alpha}+\beta\left(1-q_{i}\right)^{\alpha}} \\
\text { and } & \\
U_{\lambda}(\theta)= & \frac{2 n}{\lambda}-\frac{n}{1+\lambda}-\sum_{i=1}^{n} x_{i}+(\alpha-1) \\
& -2 \alpha \sum_{i=1}^{n} q_{i}^{(\lambda)} \frac{q_{i}^{\alpha-1}-\beta\left(1-q_{i}\right)^{\alpha-1}}{q_{i}^{\alpha}+\beta\left(1-q_{i}\right)^{\alpha}} .
\end{aligned}
$$

For interval estimation and hypothesis testing on the model parameters, we require the $3 \times 3$ observed information matrix $J=J(\theta)$ with elements given by

$$
\begin{aligned}
J_{\alpha \alpha}(\theta)= & \frac{-n}{\alpha^{2}}-2 \beta \sum_{i=1}^{n} \frac{q_{i}^{\alpha}\left(1-q_{i}\right)^{\alpha}\left[\log \left(\frac{q_{i}}{1-q_{i}}\right)\right]^{2}}{\left[q_{i}^{\alpha}+\beta\left(1-q_{i}\right)^{\alpha}\right]^{2}}, \\
J_{\alpha \beta}(\theta)= & -2 \sum_{i=1}^{n} \frac{q_{i}^{\alpha}\left(1-q_{i}\right)^{\alpha} \log \left(1-q_{i}\right)}{\left[q_{i}^{\alpha}+\beta\left(1-q_{i}\right)^{\alpha}\right]^{2}}, \\
J_{\alpha \lambda}(\theta)= & \sum_{i=1}^{n} \frac{q_{i}^{(\lambda)}}{q_{i}}-\sum_{i=1}^{n} \frac{q_{i}^{(\lambda)}}{1-q_{i}}+\sum_{i=1}^{n} \frac{q_{i}^{(\lambda)} q_{i}^{\alpha-1}\left[1+\alpha \log \left(q_{i}\right)\right]}{q_{i}^{\alpha}+\beta\left(1-q_{i}\right)^{\alpha}}-\alpha \sum_{i=1}^{n} q_{i}^{(\lambda)} \frac{q_{i}^{\alpha-1}-\beta\left(1-q_{i}\right)^{\alpha-1}}{q_{i}^{\alpha}+\beta\left(1-q_{i}\right)^{\alpha}} \\
& -\beta \sum_{i=1}^{n} \frac{q_{i}^{(\lambda)}\left(1-q_{i}\right)^{\alpha-1}\left[1+\alpha \log \left(1-q_{i}\right)\right]}{q_{i}^{\alpha}+\beta\left(1-q_{i}\right)^{\alpha}}, \\
J_{\beta \beta}(\theta)= & \frac{n}{\beta^{2}}+2 \sum_{i=1}^{n}\left[\frac{\left(1-q_{i}\right)^{\alpha}}{q_{i}^{\alpha}+\beta\left(1-q_{i}\right)^{\alpha}}\right]^{2},
\end{aligned}
$$




$$
J_{\beta \lambda}(\theta)=2 \alpha \sum_{i=1}^{n} \frac{q_{i}^{(\lambda)} q_{i}^{\alpha}\left(1-q_{i}\right)^{\alpha}}{\left[q_{i}^{\alpha}+\beta\left(1-q_{i}\right)^{\alpha}\right]^{2}},
$$

and

$$
\begin{aligned}
J_{\lambda \lambda}(\theta)= & \frac{-2 n}{\lambda^{2}}+\frac{n}{(1+\lambda)^{2}}+(\alpha-1) \sum_{i=1}^{n} \frac{q_{i}^{(\lambda \lambda)} q_{i}-\left[q_{i}^{(\lambda)}\right]^{2}}{q_{i}^{2}}+(1-\alpha) \sum_{i=1}^{n} \frac{q_{i}^{(\lambda \lambda)}\left(1-q_{i}\right)+\left[q_{i}^{(\lambda)}\right]^{2}}{\left(1-q_{i}\right)^{2}} \\
& -2 \alpha \sum_{i=1}^{n} q_{i}^{(\lambda \lambda)} \frac{q_{i}^{\alpha-1}-\beta\left(1-q_{i}\right)^{\alpha-1}}{q_{i}^{\alpha}+\beta\left(1-q_{i}\right)^{\alpha}}-2 \alpha(\alpha-1) \sum_{i=1}^{n}\left[q_{i}^{(\lambda)}\right]^{2} \frac{q_{i}^{\alpha-2}+\beta\left(1-q_{i}\right)^{\alpha-2}}{q_{i}^{\alpha}+\beta\left(1-q_{i}\right)^{\alpha}} \\
& +2 \alpha^{2} \sum_{i=1}^{n}\left\{q_{i}^{(\lambda)} \frac{q_{i}^{\alpha-1}-\beta\left(1-q_{i}\right)^{\alpha-1}}{q_{i}^{\alpha}+\beta\left(1-q_{i}\right)^{\alpha}}\right\}^{2},
\end{aligned}
$$

where

$$
\begin{aligned}
q_{i}^{(\lambda)} & =\frac{\lambda x_{i}\left(1+x_{i}\right) e_{\mathrm{e}}^{-\lambda x_{i}}}{(1+\lambda)^{2}}, \\
q_{i}^{(\lambda \lambda)} & =\frac{\lambda x_{i}\left(1+x_{i}\right)\left[1-\lambda-\lambda(1+\lambda) x_{i}\right] \mathrm{e}^{-\lambda x_{i}}}{(1+\lambda)^{3}} .
\end{aligned}
$$

Under the conditions that are fulfilled for parameters in the interior of the parameter space but not on the boundary, the asymptotic distribution of $\sqrt{n}(\widehat{\theta}-\theta)$ is $N_{3}\left(0, I(\theta)^{-1}\right)$, where $I(\theta)$ is the expected information matrix. In practice, we can replace $I(\theta)$ by the observed information matrix evaluated at $\widehat{\theta}$ (say $J(\widehat{\theta})$ ). We can construct approximate confidence intervals and confidence regions for the individual parameters and for the hazard and survival functions based on the multivariate normal $N_{2}\left(0, J(\widehat{\theta})^{-1}\right)$ distribution. Further, the likelihood ratio (LR) statistic can be used for comparing this distribution with some of its special sub-models. We can compute the maximum values of the unrestricted and restricted log-likelihoods to construct the LR statistics for testing some sub-models of the OLLMO-L distribution.

\section{Simulation}

In this section, a simulation study is conducted to examine the performance of the MLEs of OLLMO-L parameters. From Algorithm 2, we generate 1000 samples of size, n =20, 50, 100 and $\mathrm{n}=500$ of OLLMO-L model. The evaluation of estimates was based on the mean of the MLEs of the model parameters, the mean squared error (MSE) of the MLEs. The empirical study was conducted with software $\mathrm{R}$ and the results are given in Table 2 . The values in Table 2 indicate that the estimates are quite stable and, more importantly, are close to the true values for the these sample sizes. It is observed from Table 2 that the standard deviation decreases as $\mathrm{n}$ increases. The simulation study shows that the maximum likelihood method is appropriate for estimating the OLLMO-L parameters. In fact, the MSEs of the parameters tend to be closer to the true parameter values when $\mathrm{n}$ increases. This fact supports that the asymptotic normal distribution provides an adequate approximation to the finite sample distribution of the MLEs. The normal approximation can be improved by using bias adjustments to these estimators. Approximations to the their biases in simple models may be obtained analytically.

For large values of parameters, MSE values are higher and algorithm could not generate random data good enough. Hence, we did not give the results for large values. 
Table 2. Mean and MSE for the of the MLEs of the parameters of the OLLMO-L model.

\begin{tabular}{|ccc|c|ccc|ccc|}
\hline$\alpha$ & \multirow{2}{*}{$\beta$} & $\lambda$ & $n$ & \multicolumn{3}{c|}{ Mean } & \multicolumn{3}{c|}{ MSE } \\
\cline { 5 - 9 } & & & & $\alpha$ & $\beta$ & $\lambda$ & $\alpha$ & $\beta$ & $\lambda$ \\
\hline 0.5 & 0.5 & 1 & 20 & 0.538 & 0.632 & 1.34 & 0.246 & 0.217 & 0.122 \\
& & & 50 & 0.512 & 0.615 & 1.315 & 0.2407 & 0.155 & 0.103 \\
& & & 100 & 0.5093 & 0.605 & 1.170 & 0.237 & 0.148 & 0.081 \\
& & & 500 & 0.503 & 0.54 & 1.28 & 0.213 & 0.135 & 0.045 \\
\hline 1 & 0.5 & 0.5 & 20 & 1.17 & 0.564 & 0.428 & 0.037 & 0.232 & 0.349 \\
& & & 50 & 1.192 & 0.563 & 0.444 & 0.036 & 0.2205 & 0.3428 \\
& & & 100 & 1.190 & 0.532 & 0.452 & 0.032 & 0.19 & 0.335 \\
& & & 500 & 1.180 & 0.5192 & 0.478 & 0.0033 & 0.02 & 0.032 \\
\hline 1 & 1 & 1 & 20 & 1.071 & 1.14 & 1.34 & 0.027 & 0.132 & 0.245 \\
& & & 50 & 1.044 & 1.091 & 1.281 & 0.013 & 0.125 & 0.122 \\
& & & 100 & 1.029 & 1.056 & 1.171 & 0.009 & 0.12 & 0.095 \\
& & & 500 & 1.011 & 1.041 & 1.02 & 0.003 & 0.008 & 0.027 \\
\hline 1 & 0.5 & 1 & 20 & 1.017 & 0.57 & 1.054 & 0.054 & 0.212 & 0.215 \\
& & & 50 & 1.009 & 0.559 & 1.044 & 0.019 & 0.206 & 0.199 \\
& & & 100 & 0.994 & 0.55 & 1.024 & 0.0095 & 0.194 & 0.035 \\
& & & 500 & 0.998 & 0.538 & 1.024 & 0.001 & 0.184 & 0.0025 \\
\hline 1 & 1 & 2 & 20 & 0.877 & 1.116 & 2.375 & 0.035 & 0.132 & 0.245 \\
& & & 50 & 0.881 & 1.080 & 2.575 & 0.023 & 0.125 & 0.122 \\
& & & 100 & 0.919 & 0.914 & 2.143 & 0.019 & 0.12 & 0.095 \\
& & & 500 & 0.956 & 0.996 & 2.047 & 0.015 & 0.017 & 0.065 \\
\hline
\end{tabular}

\section{Applications}

In this section, we provide two applications to real lifetime data to illustrate the flexibility of the OLLMO-L model. We compare the performance of the new model with those of the odd Burr Lindley, Kumaraswamy Lindley, Marshall-Olkin Lindley, Power Lindley, and Odd log-logistic Lindley models described in Table 3. Then, the MLEs of the model parameters are determined.

Table 3. Distributions

\begin{tabular}{lll}
\hline Distribution & Abbreviation & References \\
\hline Odd Burr Lindley & OBu-L & Altun et al. \\
Kumaraswamy Lindley & Kum-L & Cakmakyapan and Ozel (2015) \\
Marshall-Olkin Lindley & MO-L & Marshall and Olkin (1995) \\
Power Lindley & PL & Ghitany et al. (2013) \\
Odd Log-logistic Lindley & OLL-L & Ozel et al. (2016) \\
Odd Log-logistic Marshal-Olkin Lindley & OLLMO-L & Proposed \\
\hline
\end{tabular}

\subsection{Application to bladder cancer patients data}

In this subsection, we provide application to a real data set to demonstrate the potentiality of the OLLMO-L distribution. First, we describe the data set and determine the MLEs (and their standard errors in parentheses) of the parameters. We consider an uncensored data set corresponding to remission times (in months) of a random sample of 128 bladder cancer patients (Lee \& Wang, 2003). 
Table 4 lists the MLEs and their standard errors of the parameters from the fitted models. In order to compare the above mentioned models with the proposed OLLMO-L model, we apply goodnessof-fit tests to verify which distribution fits better the real data set. The statistics Cramer von Mises $\left(\mathrm{W}^{*}\right)$ and Anderson Darling $\left(\mathrm{A}^{*}\right)$ are described in details in Chen \& Balakrishnan (1995). In general, the smaller the values of these statistics, the better the fit to the data. The statistics in Tables 4 clearly indicate that the OLLMO-L model provides the best fit to the data.

Table 4. The estimates of parameters and goodness-of-fit statistics for bladder cancer patients data

\begin{tabular}{lcccccc}
\hline Model & \multicolumn{7}{c}{ Parameter Estimates } & $A^{*}$ & $W^{*}$ & $-\ell$ \\
\hline$P L(\alpha, \theta)$ & 0.830 & 0.294 & & 0.703 & 0.117 & 413.354 \\
& 0.047 & 0.037 & & & & \\
\hline$M O-L(\beta, \lambda)$ & 0.110 & 0.074 & & 0.103 & 0.0153 & 409.593 \\
& 0.199 & 1.544 & 0.650 & & & \\
\hline Kum $-L(a, b, \lambda)$ & 0.886 & 0.300 & 0.466 & 0.864 & 0.144 & 414.506 \\
& 0.010 & 0.027 & 0.008 & & & \\
\hline$O L L-L(\alpha, \lambda)$ & 0.836 & 0.203 & & 1.177 & 0.197 & 416.638 \\
& 0.064 & 0.014 & & & & \\
\hline$O B u-L(\alpha, \beta, \lambda)$ & 1.009 & 0.302 & 0.480 & 0.768 & 0.128 & 414.462 \\
& 0.005 & 0.027 & 0.005 & & & \\
\hline$O L L M O-L(\alpha, \beta, \lambda)$ & 1.065 & 0.0715 & 0.064 & 0.089 & 0.013 & 408.274 \\
& 0.082 & 0.08 & 0.033 & & & \\
\hline
\end{tabular}

In order to assess whether the model is appropriate, probability density and empirical distribution functions are presented in Figure 3 for all considered distributions for bladder cancer patients data. As seen from Figure 3, the estimated OLLMO-L density for the first data is unimodal. Probability density, survival, empirical distribution functions and probability-probability (P-P) plot of the OLLMO-L model is also presented in Figure 3(right). It is clear that OLLMO-L distribution is very suitable for the bladder cancer patients data.
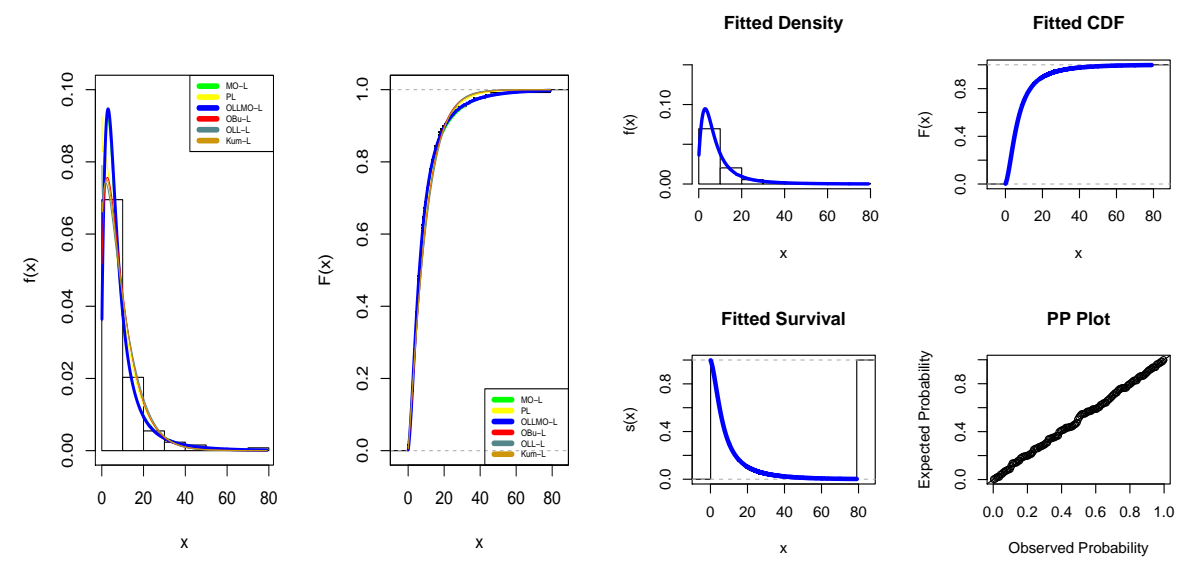

Fig. 3. Fitted density and cumulative distribution functions of the models (left) and fitted functions of OLLMO-L model (right) for bladder cancer patients data

\subsection{Application to fatigue data}

The second data set comes from the life of fatigue fracture of Kevlar 373/epoxy that are subject to constant pressure at the 90 stress level until all had failed. Hence, we have complete data with the 
exact times of failure. The data set consists of 76 observations. For the previous studies with the data sets see Andrews \& Herzberg (1985).

Table 5 provides the MLEs of the parameters for the PL, MO-L, Kum-L, OLL-L, OBu-L, and proposed OLLMO-L models. Further, we give the standard errors of the estimates and the values of $\mathrm{W}^{*}$ and $\mathrm{A}^{*}$ statistics.

Table 5. The estimates of parameters and goodness-of-fit statistics for fatigue data

\begin{tabular}{lcccccc}
\hline Model & \multicolumn{7}{c}{ Parameter Estimates } & $A^{*}$ & $W^{*}$ & $-\ell$ \\
\hline$P L(\alpha, \theta)$ & 1.142 & 0.704 & & 0.756 & 0.128 & 122.400 \\
& 0.09 & 0.081 & & & & \\
\hline$M O-L(\beta, \lambda)$ & 1.975 & 1.036 & & 0.800 & 0.136 & 122.628 \\
& 0.871 & 0.179 & & & & \\
\hline Kum $-L(a, b, \lambda)$ & 1.420 & 0.892 & 10.179 & 0.656 & 0.111 & 121.893 \\
& 0.373 & 0.925 & 0.787 & & & \\
\hline$O L L-L(\alpha, \lambda)$ & 1.259 & 0.75 & & 0.569 & 0.0964 & 121.364 \\
& 0.128 & 0.055 & & & & \\
\hline$O B u-L(\alpha, \beta, \lambda)$ & 1.342 & 0.669 & 0.943 & 0.524 & 0.089 & 121.158 \\
\hline$O L L M O-L(\alpha, \beta, \lambda)$ & 0.193 & 0.379 & 0.31 & & & \\
\hline & 1.419 & 0.338 & 0.513 & 0.493 & 0.083 & 120.019 \\
\hline
\end{tabular}

Table 5 shows that the proposed OLLMO-L model presents the smallest values of the statistics $\mathrm{W}^{*}$ and $\mathrm{A}^{*}$ among all the models. More information is provided by a usual comparison of the histograms of the fatigue data with the fitted pdfs. The fitted pdfs and cdfs are shown in Figure 4. They indicate that the OLLMO-L distribution provides more adequate fit than the other distributions. Figure 4(right) displays thr probability density, survival, empirical distribution functions and P-P plot of the OLLMO-L model. Figure 4 shows that the OLLMO-L distribution is very suitable for the bladder cancer patients data.
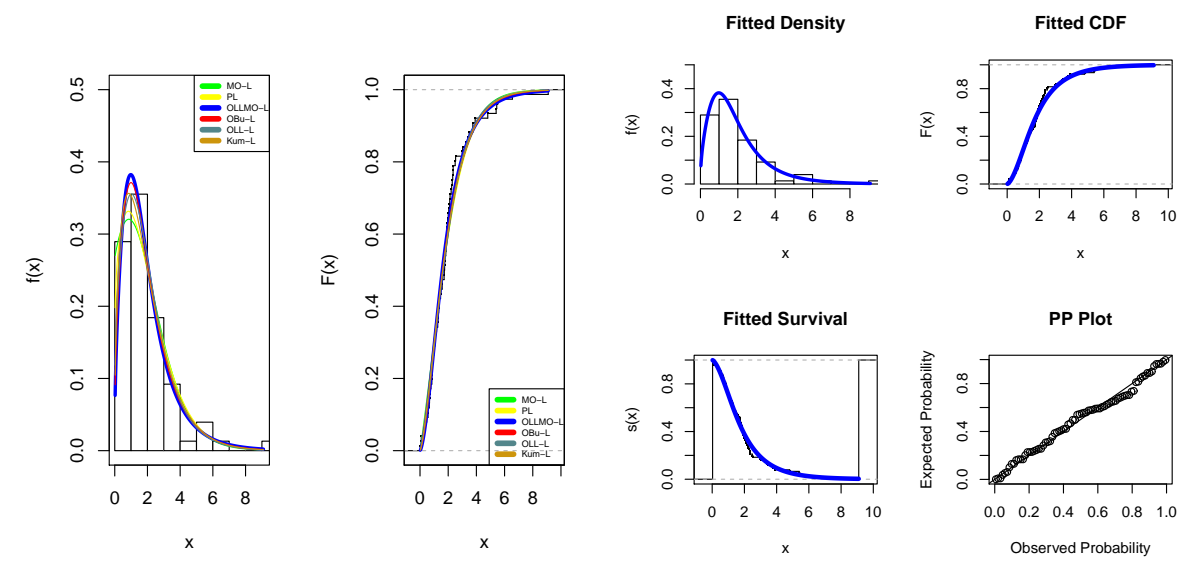

Fig. 4. Fitted density and cumulative distribution functions of the models (left) and fitted functions of OLLMO-L model (right) for fatigue data

\section{Conclusion}

In this paper, we propose the odd log-logistic Marshall-Olkin Lindley "OLLMO-L" distribution. We study some of its structural properties including an expansion for the density function and explicit 
expressions for the moments, generating function, quantile function and order statistics. The maximum likelihood method is employed for estimating the model parameters. We fit OLLMO-L distribution to two real data sets to demonstrate the usefulness of the new distribution. OLLMO-L distribution provides consistently better fits than other competing models. We hope that the proposed distribution will attract wider applications in several areas such as engineering, survival and lifetime data, hydrology and economics, among others.

\section{Acknowledgements}

The authors would like to thank the anonymous referees for their useful comments and suggestions.

\section{References}

[1] Andrews, D. F. \& Herzberg, A. M. (1985). Data: A Collection of Problems from Many Fields for the Student and Research Worker, Springer Series in Statistics, New York.

[2] Altun, G., Alizadeh, M., Altun, E. \& Ozel, G., Odd Burr Lindley distribution with properties and applications. Hacettepe Journal of Statistics and Mathematics. Forthcoming.

[3] Cakmakyapan, S. \& Ozel, G. (2014). A New Customer Lifetime Duration Distribution: The Kumaraswamy Lindley Distribution. International Journal of Trade, Economics and Finance, 5, 5, 441-444.

[4] Chen, G. \& Balakrishnan, N. (1995). A general purpose approximate goodness-of-fit test. Journal of Quality Technology, 27, 154-161.

[5] Corless, R.M., Gonnet, G.H., Hare, D.E.G., Jeffrey, D.J. \& Knuth. D.J. (1996). On the Lambert W function. Adv. Comput. Math, 5, 329-359.

[6] Cordeiro, G.M.,Alizadeh, M., Tahir, M. H., Mansoor, M., Bourguignon, M., \& Hamedani, G.G. (2015). The beta odd log-logistic generalized family of distributions, Hacettepe Journal of Mathematics and Statistics, 45, 73, 126-139.

[7] Doornik, J.A. (2007). Object-Oriented Matrix Programming Using Ox. Timberlake Consultants Press and Oxford, London, UK, third edition.

[8] Ghitany, M.E., Al-Mutairi, D.K., Balakrishhnan, N. \& (2013). Al-Enezi, L.J. Power Lindley distribution and associated inference. Computational Statistics and Data Analysis, 64, 20-33.

[9] Glänzel, W., A characterization theorem based on truncated moments and its application to some distribution families, Mathematical Statistics and Probability Theory (Bad Tatzmannsdorf, 1986), Vol. B, Reidel, Dordrecht, 1987, 75-84.

[10] Glänzel, W., Some consequences of a characterization theorem based on truncated moments, Statistics: A Journal of Theoretical and Applied Statistics, 21 (4), 1990, 613-618.

[11] Gleaton, J.U., \& Lynch, J.D. (2006). Properties of generalized log-logistic families of lifetime distributions. Journal of Probability and Statistical Science, 4, 51-64.

[12] Gleaton, J. U., \& Lynch, J. D. (2010). Extended Generalized Log-logistic Families of Lifetime Distributions with an Application. Journal of Probability and Statistical Science, 1.

[13] Gradshteyn, I.S., Ryzhik, I.M. (2000). Table of integrals, series, and products. Academic Press, San Diego.

[14] Jodrá, J. (2010). Computer generation of random variables with Lindley or Poisson-Lindley distribution via the Lambert W function. Mathematics and Computers Simulation, 81, 851-859.

[15] Lee, E. T. \& Wang, J. W. (2003). Statistical Methods for Survival Data Analysis (3rd ed.). New York: Wiley.

[16] Lindley, D.V. (1958). Fiducial distributions and Bayesian theorem. Journal of the Royal Statistical Society B, 20, 102-107.

[17] Marshall, A. W., \& Olkin, I. (1997). A new method for adding a parameter to a family of distributions with application to the exponential and Weibull families. Biometrika, 84(3), 641-652.

[18] Nadarajah, S., Bakouch, H. S. \& Tahmasbi, R. (2011). A generalized Lindley distribution. Sankhya B, 73, 331-359. 
[19] Ozel, G., Alizadeh, M., Cakmakyapan, S., Hamedani, G., Ortega, E. M. M., Cancho, G. (2016). The Odd Log-Logistic Lindley Poisson Model for Lifetime Data. Communications in Statistics: Theory and Methods, Online Published.

[20] Shanker, R. \& Mishra, A. (2013). A Quasi Lindley Distribution. African Journal of Mathematics and Computer Science Research, 6, 4, 6471.

[21] Sharma V, Singh S, Singh U. \& Agiwal V. The inverse Lindley distribution: a stress-strength reliability model with applications to head and neck cancer data. Journal of Industrial and Production Engineering (2015). 32, 3, 162173.

\section{Appendix A.}

Theorem A.1. Let $(\Omega, \mathscr{F}, \mathbf{P})$ be a given probability space and let $H=[a, b]$ be an interval for some $d<b(a=-\infty, b=\infty$ might as well be allowed $)$. Let $X: \Omega \rightarrow H$ be a continuous random variable with the distribution function $F$ and let $q_{1}$ and $q_{2}$ be two real functions defined on $H$ such that

$$
\mathbf{E}\left[q_{2}(X) \mid X \geq x\right]=\mathbf{E}\left[q_{1}(X) \mid X \geq x\right] \eta(x), \quad x \in H,
$$

is defined with some real function $\eta$. Assume that $q_{1}, q_{2} \in C^{1}(H), \eta \in C^{2}(H)$ and $F$ is twice continuously differentiable and strictly monotone function on the set $H$. Finally, assume that the equation $\eta q_{1}=q_{2}$ has no real solution in the interior of $H$. Then $F$ is uniquely determined by the functions $q_{1}, q_{2}$ and $\eta$, particularly

$$
F(x)=\int_{a}^{x} C\left|\frac{\eta^{\prime}(u)}{\eta(u) q_{1}(u)-q_{2}(u)}\right| \exp (-s(u)) d u,
$$

where the function $s$ is a solution of the differential equation $s^{\prime}=\frac{\eta^{\prime} q_{1}}{\eta q_{1}-q_{2}}$ and $C$ is the normalization constant, such that $\int_{H} d F=1$. 